\title{
Constraints on the flux of ultra-high energy neutrinos from Westerbork Synthesis Radio Telescope observations
}

\author{
S. Buitink ${ }^{1,2}$, O. Scholten ${ }^{3}$, J. Bacelar ${ }^{4}$, R. Braun 5 , A. G. de Bruyn ${ }^{6,7}$, H. Falcke ${ }^{2,7}$, K. Singh ${ }^{3}$, B. Stappers ${ }^{8}$, \\ R. G. Strom ${ }^{7,9}$, and R. al Yahyaoui ${ }^{3}$
}

1 Lawrence Berkeley National Laboratory, Berkeley, CA 94720, USA e-mail: sbuitink@lbl.gov

2 Department of Astrophysics, IMAPP, Radboud University, 6500 GL Nijmegen, The Netherlands

3 Kernfysisch Versneller Instituut, University of Groningen, 9747 AA Groningen, The Netherlands

4 ASML Netherlands BV, PO Box 324, 5500 AH Veldhoven, The Netherlands

5 CSIRO - Astronomy and Space Science, PO Box 76, Epping NSW 1710, Australia

${ }^{6}$ Kapteyn Institute, University of Groningen, 9747 AA Groningen, The Netherlands

7 ASTRON, 7990 AA Dwingeloo, The Netherlands

8 School of Physics \& Astronomy, Alan Turing Building, Univ. of Manchester, Manchester M13 9PL, UK

9 Astronomical Institute A. Pannekoek, University of Amsterdam, 1098 SJ, The Netherlands

Received 20 January 2010 / Accepted 18 February 2010

\begin{abstract}
Context. Ultra-high energy (UHE) neutrinos and cosmic rays initiate particle cascades underneath the Moon's surface. These cascades have a negative charge excess and radiate Cherenkov radio emission in a process known as the Askaryan effect. The optimal frequency window for observation of these pulses with radio telescopes on the Earth is around $150 \mathrm{MHz}$.

Aims. By observing the Moon with the Westerbork Synthesis Radio Telescope array we are able to set a new limit on the UHE neutrino flux.

Methods. The PuMa II backend is used to monitor the Moon in 4 frequency bands between 113 and $175 \mathrm{MHz}$ with a sampling frequency of $40 \mathrm{MHz}$. The narrowband radio interference is digitally filtered out and the dispersive effect of the Earth's ionosphere is compensated for. A trigger system is implemented to search for short pulses. By inserting simulated pulses in the raw data, the detection efficiency for pulses of various strength is calculated.

Results. With 47.6 hours of observation time, we are able to set a limit on the UHE neutrino flux. This new limit is an order of magnitude lower than existing limits. In the near future, the digital radio array LOFAR will be used to achieve an even lower limit.
\end{abstract}

Key words. astroparticle physics - neutrinos - methods: data analysis - methods: observational - radiation mechanisms: non-thermal

\section{1. introduction}

The cosmic ray energy spectrum follows a power law distribution extending up to extremely large energies. At the Pierre Auger Observatory (PAO) cosmic rays (CRs) are observed up to energies around $\sim 10^{20} \mathrm{eV}$. Above the Greisen-Zatsepin-Kuzmin $(\mathrm{GZK})$ energy of $6 \times 10^{19} \mathrm{eV}$, CRs can interact with the cosmic microwave background photons. In the most efficient interaction, a $\Delta$-resonance is produced which decays into either a proton and a neutral pion or a neutron and a positively charged pion. Charged pions decay and produce neutrinos. The energy loss length for $\Delta$-resonance production is $\sim 50 \mathrm{Mpc}$ (Greisen 1966; Zatsepin \& Kuzmin 1966).

Recent results of the PAO have confirmed a steepening in the cosmic ray spectrum at the GZK energy (Abraham 2008). This steepening is not necessarily a clear cut-off, as CRs from local sources may arrive at Earth with super-GZK energies. Because of their large energies, these particles will only deflect slightly in the (extra-) Galactic field during their propagation, and their arrival directions correlate with their sources (Abraham 2007).

Sources at distances larger than $50 \mathrm{Mpc}$ can be found by observing neutrinos that are produced in GZK interactions. Since neutrinos are chargeless they will propagate in a straight line from the location where the GZK interaction took place to the observer, thus conserving the directional information. In addition, while CRs from distant sources pile up at the GZK energy, information about the CR spectrum at the source is conserved in the GZK neutrino flux. Other possible sources of UHE neutrinos are decaying supermassive dark matter particles or topological defects. This class of models is refered to as top-down models (see for example Stanev 2004, for a review).

Because of their small interaction cross section and low flux, the detection of cosmic neutrinos calls for extremely large detectors. Assuming the Waxman-Bahcall flux (Waxman \& Bahcall 1998a; Bahcall \& Waxman 2001), even at low energies in the $\mathrm{GeV}$ range, the flux is not higher than a few tens of neutrinos per $\mathrm{km}^{2}$ per year. Kilometer-scale detectors are not easily built but can be found in nature. For example, interaction of neutrinos in ice or water can be detected by the Cherenkov light produced by the lepton track or cascade. The nearly completed IceCube detector (Ahrens 2003) will cover a km³ volume of South Pole ice with optical modules, while Antares (Aslanides 1999) and its successor KM3NET (Katz 2006) exploit the same technique in the Mediterranean sea. Even larger volumes can be covered by observing large detector masses from a distance. The ANITA balloon mission (Barwick 2006) monitors an area of a million $\mathrm{km}^{2}$ of South Pole ice from an altitude of $\sim 37 \mathrm{~km}$ and the 
FORTE satellite (Lehtinen et al. 2004) can pick up radio signals coming from the Greenland ice mass. Alternatively, cosmic ray experiments like the Pierre Auger Observatory can possibly distinguish cosmic ray induced air showers from neutrino induced cascades at very high zenith angles where the atmosphere is thickest and only neutrinos can interact close to the detector.

The Moon offers an even larger natural detector volume. When CRs or neutrinos hit the Moon they will interact with the medium. CRs will start a particle cascade just below the Lunar surface, while neutrinos will interact deeper inside the Moon, also creating a hadronic shower. The negative charge excess of a particle cascade inside a dense medium will cause the emission of coherent Cherenkov radiation in a process known as the Askaryan effect (Askaryan 1962). This emission mechanism has been experimentally verified at accelerators (Saltzberg 2001; Gorham 2000) and extensive calculations have been performed to quantify the effect (Zas et al. 1992; Alvarez-Muñiz \& Zas 1997). The idea to observe this type of emission from the Moon with radio telescopes was first proposed by Dagesamanskii \& Zheleznyk (1989) and the first experimental endeavours in this direction were carried out with the Parkes telescope (Hankins et al. 1996), at Goldstone (GLUE) (Gorham 2004), and with the Kalyazin Radio Telescope (Beresnyak et al. 2005). LUNASKA (Lunar UHE Neutrino Astrphysics with the Square Kilometer Array) is a project that is currently performing lunar Cherenkov measurements with ATCA (the Australia Telescope Compact Array) with a $600 \mathrm{MHz}$ bandwidth at $1.2-1.8 \mathrm{GHz}$ (James et al. 2009).

Falcke \& Gorham (2003) suggested to use low-frequency telescopes (like LOFAR) for such an experiment. It is shown by Scholten (2006) that observing at lower frequencies has the distinct advantage that radio pulses have a much higher chance of reaching the observer, as will be explained in the next section. In this work we use data recorded with the Westerbork Synthesis Radio Telescope (WSRT) in the frequency range of 113-168 MHz to set a new limit on the flux of UHE neutrinos. A first reporting of this limit is made in (Scholten et al. 2009).

\section{Detection principle}

UHE neutrinos or CRs interact below the lunar surface. In the case of a CR, all energy is converted into a hadronic shower. In a neutrino interaction, only about $20 \%$ of the energy is converted into a hadronic shower, while the other $80 \%$ is carried off by a lepton (corresponding to the neutrino flavor), which will not produce any observable radio emission. Muons will not produce enough charge density, while electromagnetic showers become elongated at energies above $E_{\mathrm{LPM}}=10^{18} \mathrm{eV}$ due to the Landau-Pomeranchuk-Migdal (LPM) effect (Alvarez-Muñiz $\&$ Zas 1998). For these showers the angular spread of the radio emission around the Cherenkov angle becomes very small, severely lowering the chance of detection.

For proton energies exceeding $10^{20} \mathrm{eV}$ it is predicted that the LPM effect will start to play a role since many of the leptons and photons which are created as secondary particles have energies in excess of the $E_{\mathrm{LPM}}$. This has the effect of creating a lopsided hadronic shower with a rather long "tail" (Alvarez-Muñiz \& Zas 1998). The bulk of the charged particles in the shower is still present over a length which one would have obtained ignoring the LPM effect and our estimates should thus apply also to the hadronic part of showers initiated by neutrinos of energies ranging up to $10^{23} \mathrm{eV}$.

The lateral size of the cascade is of the order of $10 \mathrm{~cm}$ so the radio emission is coherent up to $\sim 3 \mathrm{GHz}$. Former experiments, like GLUE, have observed at high frequencies $(2.2 \mathrm{GHz})$ where the emission is strongest. For lower frequencies, however, the angular spread of the emission around the Cherenkov angle increases due to diffraction. For emission at the Cherenkov angle, only those showers can be observed that hit the rim of the Moon, under such an angle that the emission will not be internally reflected at the Lunar surface. With a larger angular spread in the emission a wider range of geometries is allowed and a larger part of the lunar surface acts as a radiation source. When the wavelength is of the order of the shower length, several meters, the emission becomes nearly isotropic and pulses can be expected to come from the whole Moon (Scholten 2006). In our experiment we exploit this optimal frequency range around $150 \mathrm{MHz}$.

The intensity of the radio emission from a hadronic shower with energy $E_{\mathrm{s}}$ in the lunar regolith can be parameterized as (Zas et al. 1992; Alvarez-Muñiz \& Zas 1997; Scholten 2006)

$$
\begin{aligned}
F\left(\theta, v, E_{\mathrm{s}}\right)= & 3.86 \times 10^{4} \mathrm{e}^{-Z^{2}}\left(\frac{\sin \theta}{\sin \theta_{\mathrm{c}}}\right)^{2}\left(\frac{E_{\mathrm{s}}}{10^{20} \mathrm{eV}}\right)^{2}\left(\frac{d_{\mathrm{moon}}}{d}\right)^{2} \\
& \times\left(\frac{v}{v_{0}\left(1+\left(v / v_{0}\right)^{1.44}\right)}\right)^{2}\left(\frac{\Delta v}{100 \mathrm{MHz}}\right) \mathrm{Jy}
\end{aligned}
$$

with

$$
Z=(\cos \theta-1 / n)\left(\frac{n}{\sqrt{n^{2}-1}}\right)\left(\frac{180}{\pi \Delta_{c}}\right),
$$

where $\Delta v$ is the bandwidth, $v$ the central frequency and $v_{0}=$ $2.5 \mathrm{GHz}$. The average Earth-Moon distance $d_{\text {moon }}=3.844 \times$ $10^{8} \mathrm{~m}, d$ is the distance to the observer. The Cherenkov angle is given by $\cos \theta_{c}=1 / n$, where $n$ is the index of refraction and $\theta$ is the angle under which radiation is emitted relative to the direction of shower propagation. The spread of radiation around the Cherenkov angle is given by

$\Delta_{\mathrm{c}}=4.32^{\circ}\left(\frac{1}{v[\mathrm{GHz}]}\right)\left(\frac{L\left(10^{20} \mathrm{eV}\right)}{L\left(E_{\mathrm{s}}\right)}\right)$,

where $L$ is the shower length depending on primary energy.

The regolith is the top layer of the Moon and consists of dust and small rocks. The properties of this layer are known from samples brought from the Moon by the Apollo missions (Olhoeft \& Strangway 1975). The average index of refraction is $n=1.8$ and the mean attenuation length is found to be $\lambda_{\mathrm{r}}=(9 / v[\mathrm{GHz}]) \mathrm{m}$ for radio waves (Olhoeft \& Strangway 1975; Heiken et al. 1991). There are sizable differences in, especially, the reported values of the attenuation length. The effects of this uncertainty on the extracted limits is discussed in Sect. 7. The thickness of the regolith is known to vary over the lunar surface. At some depth there is a (probably smooth) transition to solid rock, for which the density is about twice that of the regolith. Wieczorek \& Zuber (2001) report that the density is almost homogeneous down to a depth of $20 \mathrm{~km}$. In Scholten (2006) the effects of pure rock and regolith are simulated and found to give very similar detection limits for low frequencies.

As the radiation leaves the Moon it refracts through the surface. In Gorham (2004) and James \& Protheroe (2009) the effects of this refraction for smooth and irregular surfaces are described. It is shown that the angular spread $\Delta \theta$ increases due to this refraction and that this effect is especially strong when the angle at which the radiation approaches the lunar surface is close to the angle of total internal reflection. The larger angular spread increases the acceptance but also increases the energy threshold for detection since the radiated power spreads out over a larger 
area. Small scale irregularities of the lunar surface make this effect stronger because variations in the surface tangent within the radiation beam will increase the angular spread by refraction even more. At the frequencies at which we observe, these effects are of less importance since $\Delta \theta$ is already large at the source due to diffraction and thus the increase due to surface irregularities can safely be ignored.

\section{Detection with WSRT}

The Westerbork Radio Synthesis Telecope (WSRT) is an array telescope consisting of 14 parabolic telescopes of $25 \mathrm{~m}$ on a $2.7 \mathrm{~km}$ east-west line. The NuMoon experiment uses the Low Frequency Front Ends (LFFEs) which cover the frequency range 115-180 MHz. Each LFFE records full polarization data. For our observations we use the Pulsar Machine II (PuMa II) backend (Karuppusamy et al. 2006), which can record a maximum bandwidth of $160 \mathrm{MHz}$, sampled as 8 subbands of $20 \mathrm{MHz}$ each.

Only 11 of the 12 equally spaced WSRT dishes are used for this experiment which means that when the telescopes are added in phase the resultant beam on the sky is a fan beam (Jansen 2009). The phases required to add the dishes coherently are determined by observations of a known calibrator source, which at these frequencies is Cassiopeia A. Adjusting the phase relations between the 8 subbands they can be pointed to any location within the primary beam of the $25 \mathrm{~m}$ dish.

We use two beams of 4 bands each, centered around 123, 137, 151, and $165 \mathrm{MHz}$. The two beams are aimed at different sides of the Moon, each covering about one third of the lunar surface, in order to enlarge the effective aperture and create the possibility of an anti-coincidence trigger. A lunar Cherenkov pulse should only be visible in one of the two beams. Because of overlap in the subbands the total bandwidth per beam is $65 \mathrm{MHz}$. The system has a real time automatic gain control (AGC) system, that stabilizes the average gain of the output signal.

For each subband, the time series data is recorded at several storage nodes with a sampling frequency of $40 \mathrm{MHz}$.

The data is processed in blocks of $0.1 \mathrm{~s}$, each block being divided in 200 traces of 20000 time samples. The signal of individual WSRT dishes is 2 bit, limiting the dynamic range of an 11-dish observation to 34 . We will discuss the implications of this limited dynamic range in Sect. 4. There is data for two beams, each containing 4 frequency bands and 2 polarization directions.

The data analysis is performed in the following steps:

- RFI background reduction. Radio Frequency Interference (RFI) is narrow band anthropogenic emission, which can be responsible for a large part of the received power and has to be filtered out of the data. For all time traces an FFT is produced and for each data block the 200 frequency spectra are added to obtain an integrated frequency spectrum. The baseline of this spectrum is fitted with a 9th order polynomial function and bins containing a value exceeding the fit by $50 \%$ are marked as RFI lines. In each individual frequency spectrum all bins that are marked as RFI lines are set to zero. This procedure is carried out separately for each of the 4 frequency bands and the 2 polarizations. The number of RFI lines per spectrum varies with time and is different for all frequency bands and polarizations, but does seldom exceed 200 . The corresponding loss in bandwidth is $\sim 2 \%$ at maximum. Figure 1 shows frequency spectra of $10 \mathrm{~s}$ of data before RFI removal. In the highest frequency band the upper end of the spectrum is suppressed by a band pass filter, lowering
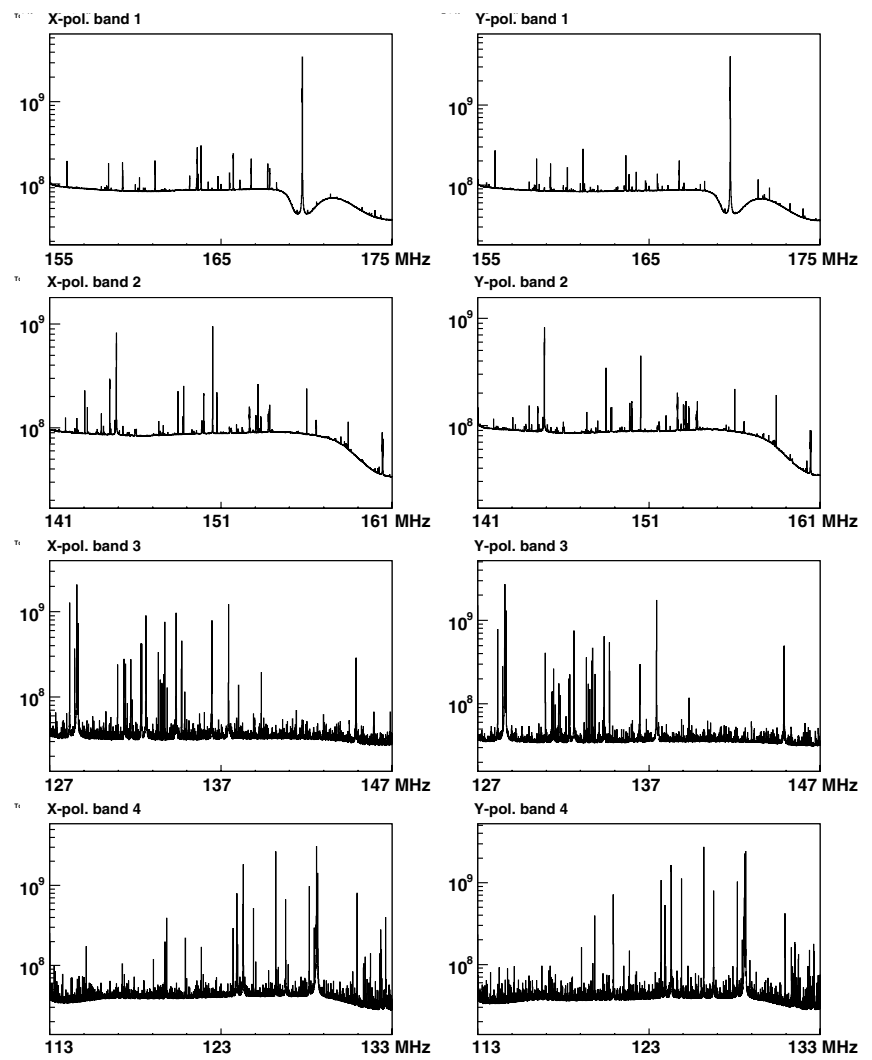

Fig. 1. Frequency spectra of $10 \mathrm{~s}$ of data for all bands and polarizations. The narrow RFI lines that exceed a fit to the curve by $50 \%$ are put to zero.

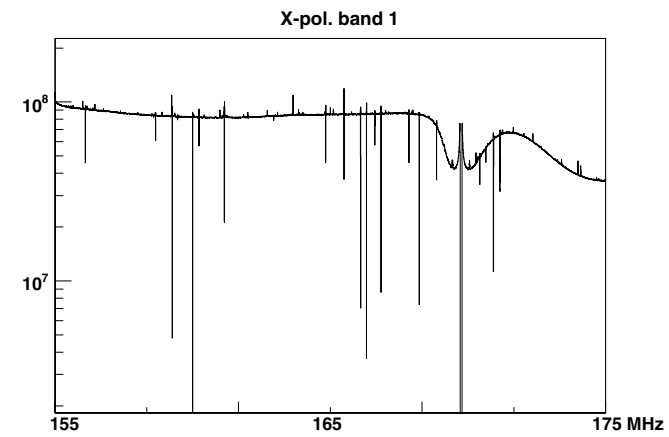

Fig. 2. Frequency spectra of $10 \mathrm{~s}$ of data for band 1 and $x$ polarization after RFI removal. The spectrum is composed of 100 spectra that have been subjected to RFI removal separately. In each spectrum a frequency sample is set to zero when the amplitude exceeds the fitted background curve by more than $50 \%$. When a certain sample contains an RFI line in all 100 spectra, it has value zero in this integrated spectra. Samples that have a non-zero value below the background curve correspond to RFI lines that are only present in part of the 100 spectra.

the effective bandwidth. In other frequency bands a similar suppression can be seen, but this is compensated by the overlap between the different bands. Our effective bandwidth is $55 \mathrm{MHz}$, ranging from 113 to $168 \mathrm{MHz}$. An example of a ten second frequency spectrum after RFI removal is shown in Fig. 2.

- Ionospheric de-dispersion. After the RFI removal the data is still in the frequency domain. The de-dispersion is performed by applying a frequency dependent phase shift to the data before transforming back to the time domain. The Vertical TEC values, that are needed for the de-dispersion, are provided by the DLR Institut für Kommunikation und 
Navigation ${ }^{1}$. These were used to calculate the STEC by compensating for the Moon elevation. Because of variations in the thickness of the ionosphere on short timescales and over distance, we assume the presence of an error in the dedispersion, resulting in an increased time width of the pulses and an offset between the arrival times of pulses in different frequency bands. The implications hereof for our analysis are further discussed in Sect. 4.

- Evaluation of $\boldsymbol{P}_{\mathbf{5}}$. After de-dispersion, an inverse FFT is performed to transform the data back into the time domain. The cutting of RFI lines increases the noise level in the time samples close to the edges of the time trace. The de-dispersion can move this increased noise further backward in time. To avoid triggering on this noise the first and last 250 time samples are excluded from analysis, corresponding to $0.25 \%$ of the observation time. Next, we calculate $P_{5}$, the power integrated over 5 consecutive samples normalized over one trace

$$
P_{5}=\frac{\sum_{\text {samples }} P_{x}}{\left\langle\sum_{5 \text { samples }} P_{x}\right\rangle}+\frac{\sum_{5 \text { samples }} P_{y}}{\left\langle\sum_{5 \text { samples }} P_{y}\right\rangle},
$$

where the averaging is done over one time trace (20000 time samples), and $x$ and $y$ denote the two polarizations. The integration has been chosen to be over 5 samples, because this is the typical number of samples over which the power is spread for a bandwidth limited and Nyquist sampled pulse with a small dispersion (see Appendix A).

- Pulse search. The data is scanned for values of $P_{5}$ exceeding 5. The meaning of this threshold can be understood from Eq. (4). If we define $\sigma^{2}$ as the mean power in one time sample (assuming for simplicity that it is equal for the $x$ - and $y$-polarizations), then the trigger condition can be written as

$$
\sum_{5 \text { samples }} P_{x}+\sum_{5 \text { samples }} P_{y}>25 \sigma^{2},
$$

meaning that the total power in ten bins (five in the $x$ polarization and five in the $y$ polarization) must add up to a value larger than $25 \sigma^{2}$, where the average is $10 \sigma^{2}$. The band with the highest frequency is first scanned for $P_{5}$ values exceeding 5. When such a value is found the $P_{5}$ values of the other 3 frequency bands are evaluated near this position. A time offset between pulses in the different bands of

$$
\Delta t=1.34 \times 10^{9} \times 0.30 \times \operatorname{STEC}\left(\frac{1}{v_{1}^{2}}-\frac{1}{v_{2}^{2}}\right)
$$

is allowed based on an error of $30 \%$ on the STEC value. When a $P_{5}$ value exceeding 5 is found in all bands the time trace is permanently stored, together with information on the RFI lines and the data of the corresponding time trace in the other beam. No search is done for a second pulse in the same trace. An estimation of the resulting loss in effective observation time is given in Sect. 5. For each trigger the location, maximum value, width and offsets between locations in the different bands are stored. The width is defined as the number of consecutive $P_{5}$ values that exceed 5 . The value $S$ is defined as the sum over the maximum $P_{5}$ values in the 4 frequency bands

$$
S=\sum_{4 \text { bands }} P_{5}
$$

1 http://www.dlr.de/kn

\section{Observations}

Table 1 contains a list of the 22 observation runs performed between June 9, 2007 and November 11, 2008. The number of raw triggers per hour is much higher than usual for the runs of June 9, 2007 and June 7/8, 2008. The top panel in Fig. 3 shows the number of triggers per data block for one hour of one of these runs. The number of triggers is only plotted when it exceeds 20 . The maximum number of triggers per block is equal to the number of traces: 200. This maximum is often reached, which means the run is not reliable. The bottom panel shows the number of triggers per block for one hour of data of a regular observation period. The observation runs for which the number of triggers per data block is exceptionally high for a long period of time have been excluded from the analysis.

Figure 4 shows the distribution of $S$ for the triggered events. The top curve corresponds to the raw triggers. Several additional cuts are applied:

- Timer signal (T) The data contains short strong pulses that repeat at a regular interval. They can be visualized by plotting a distribution of pulse times folded by an appropriate time interval. Figure 5 shows the number of triggers in $10 \mathrm{~s}$ of data against the number of the time sample folded by 390625 . This corresponds to a frequency of $102.4 \mathrm{~s}^{-1}$.

The specific time interval of these pulses suggest a technical origin. Cutting out the time intervals in which these pulses occur corresponds to a loss of $\sim 10 \%$ of observation time.

- $\boldsymbol{P}_{\mathbf{5}}$ width $(\mathbf{W})$. We define the width of a pulse $W$ as the number of consecutive $P_{5}$ values exceeding the threshold. For a real lunar pulse, $W$ should be limited. However, for increasingly tighter cuts on $W$, the probability of excluding a proper pulse grows. The value for the cut on $W$ was determined by examining the efficiency for recovering pulses in a simulation, as is explained in Sect. 4, showing a recovery rate of over $80 \%$ by choosing $W<12$ for all four frequency bands. Since this value is not obtained by optimizing the distribution of $S$ we have avoided to introduce a bias by following this procedure.

- Anti-coincidence (A). A lunar pulse should be visible in only one of the two beams. An anti-coincidence trigger is set up by excluding events for which a pulse was found in both beams in the same time trace.

Figure 4 displays distributions of $S$ after application of only the timer cut (T), the timer and width cut (TW), and a combination of all cuts (TWA). The line enclosing the black area in Fig. 4 corresponds to the distribution of triggers that are expected if the background is pure Gaussian noise (see Appendix B for details of the calculation). After all cuts have been applied the number of triggers for which $S>23$ is a factor of 3-4 higher than the amount of triggers expected for Gaussian noise, while the largest $S$ value in the distribution is about three times as large as the highest $S$ value for Gaussian noise. Apparently, the background includes pulsed noise that produces triggers and contains pulses that are narrow enough to survive the cut on width. The properties of these pulses are further explored in Sect. 8. Due to the pulsed noise, the limit that we derive for the neutrino flux is less stringent than estimated in Scholten (2006), where the existence of this background was not anticipated.

For reference, Fig. 6 shows the difference between the tails of the distribution of $S$ for a $W<10$ and a $W<12$ cut. In the latter case the highest value of $S$ is larger, but the corresponding decrease in detection efficiency makes this cut unfavorable (see Sect. 4) 
Table 1. Observation runs.

\begin{tabular}{|c|c|c|c|c|c|}
\hline Date & Hours & STEC (lo/hi) & No. raw triggers & No. triggers after cuts $(S>23)$ & No. triggers Gaussian noise $(S>23)$ \\
\hline 2007 Jun. $9^{a}$ & $4.7^{b}$ & $11.8 / 16.6$ & 200427 & 49679 & 8943 \\
\hline 2007 Sep. 21 & 2 & $15.6 / 19.5$ & 668917 & 26812 & 13128 \\
\hline 2008 Jan. 13 & $1.3^{b}$ & $18.0 / 24.3$ & 119032 & 6951 & 6001 \\
\hline 2008 Jun. $7^{a}$ & 4.25 & $11.7 / 17.5$ & 1961907 & 170672 & 21752 \\
\hline 2008 Jun. $8^{a}$ & 5 & $9.8 / 11.3$ & 1313378 & 80140 & 12815 \\
\hline 2008 Aug. 24 & 5 & $3.5 / 7.5$ & 792979 & 36314 & 4029 \\
\hline 2008 Aug. 29 & 3 & $6.5 / 7.0$ & 563692 & 45214 & 3331 \\
\hline 2008 Aug. 29 & 2 & $8.0 / 8.3$ & 602049 & 29554 & 4317 \\
\hline 2008 Aug. $29^{a}$ & 4.8 & $6.5 / 9.7$ & 1719443 & 96998 & 7425 \\
\hline 2008 Sep. 2 & 5 & $12.8 / 15.3$ & 880508 & 51329 & 18937 \\
\hline 2008 Sep. 16 & 3.75 & $5.5 / 11.0$ & 233192 & 23733 & 6616 \\
\hline 2008 Sep. 16 & 5 & $5.9 / 7.2$ & 163819 & 20138 & 4841 \\
\hline 2008 Sep. 21 & 4.5 & $3.3 / 4.8$ & 244276 & 27388 & 3451 \\
\hline 2008 Sep. $21^{a}$ & 5 & $4.0 / 12.8$ & 1282457 & 76573 & 9728 \\
\hline 2008 Sep. 28 & 3.7 & $10.5 / 12$ & 65725 & 67910 & 10345 \\
\hline 2008 Sep. 28 & 3.8 & $11.6 / 13.7$ & 622598 & 47580 & 11958 \\
\hline 2008 Oct. 14 & 4.5 & $5.9 / 9.9$ & 566611 & 58127 & 6531 \\
\hline 2008 Oct. 14 & 4.5 & $5.9 / 7.4$ & 217113 & 30346 & 4165 \\
\hline 2008 Nov. 11 & 3.7 & $3.5 / 7.4$ & 941369 & 27160 & 2624 \\
\hline Total $^{c}$ & 51.1 & $3.3 / 24.3$ & 6681880 & 430646 & 100274 \\
\hline
\end{tabular}

Notes. ${ }^{(a)}$ Excluded from analysis due to exceptional amount of raw triggers.

(b) Only single beam data available.

${ }^{(c)}$ Not counting excluded runs.
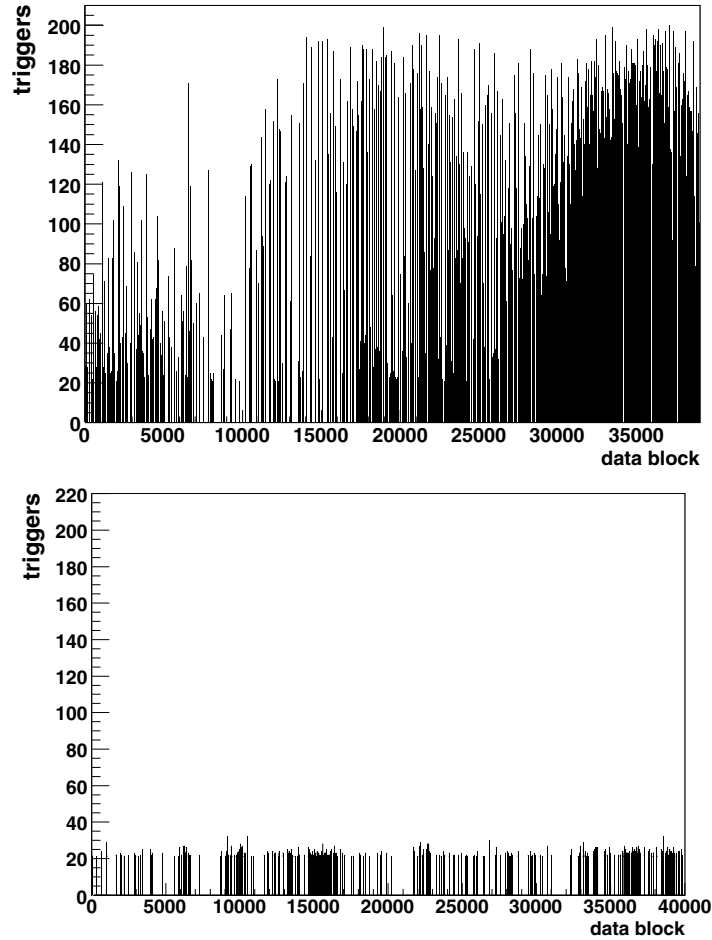

Fig. 3. Number of triggers per block is plotted against the number of the data block for one hour of data of the June 7, 2008 observation (top) and for one hour of the second observations of August 29, 2008 (bottom). Only values exceeding 20 are plotted. The maximum value of 200 indicates completely saturated data.

\section{Simulations}

\subsection{Pulse recovery}

The efficiency with which pulses are found by the analysis procedure and the effects of data cuts and ionospheric dispersion are

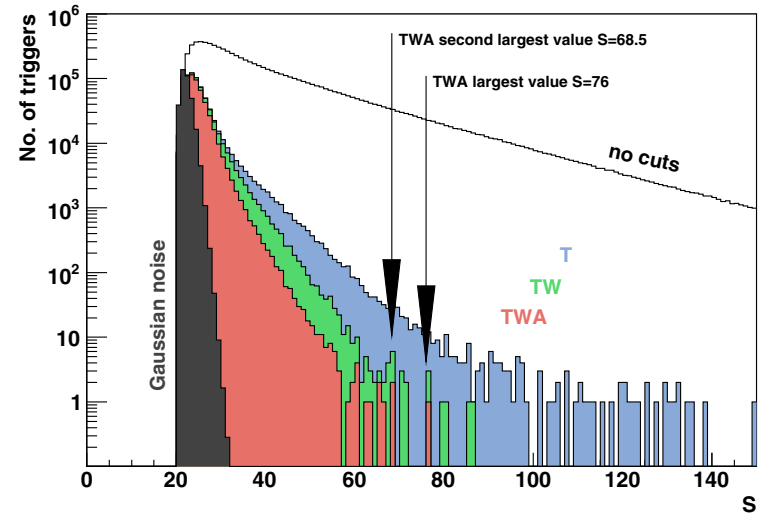

Fig. 4. Distribution of $S$ for several cuts. The top curve represents the distribution of raw triggers. The other distribution represent, in order of decreasing number of contained events, the T, TW, and TWA cut. The black area corresponds to the distribution of triggers that are expected for a background of pure Gaussian noise.

simulated by adding pulses to raw data. The received power and the power after RFI reduction are different for all bands and polarizations and change with time due to the dynamic gain matching in the electronics of WSRT. To correct for this the pulses are normalized following the same procedure as in the analysis described in the previous sections i.e. the pulse strength, denoted by $S_{i}$, is expressed in dimensionless units as defined in Eq. (7). These pulses are delta peaks inserted at random times with a random phase. Because the pulses are band width limited, the bulk of the power in such a pulse typically spreads out over a few time samples (see Appendix A). The pulse is dispersed corresponding to a particular TEC value, named simTEC, and the amplitudes are rounded off towards nearest integer within the dynamic range, after adding it to the raw (i.e. before RFI mitigation) data. For the simulations we have inserted 1000 pulses in a few different $10 \mathrm{~s}$ segments of raw WSRT data. 


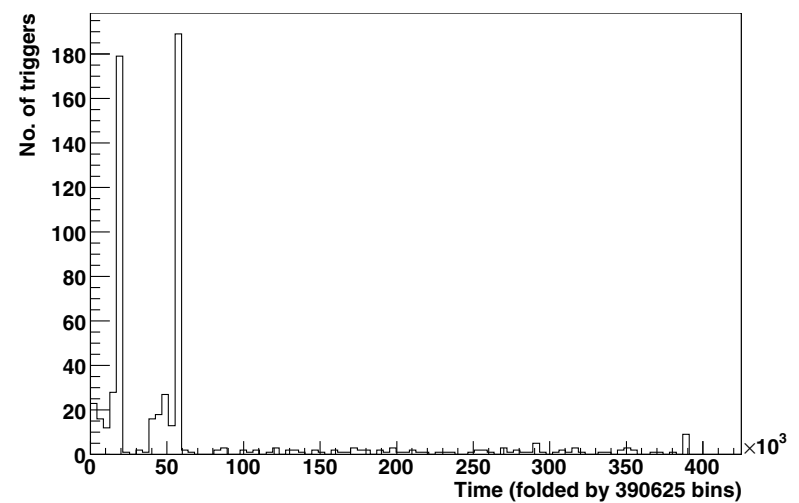

Fig. 5. Number of triggers vs. time sample folded by 390625 samples. With this folding many triggers occur at the same time, probably having a local technical origin. Triggers that occur at the positions of the peaks are excluded in the timer cut.

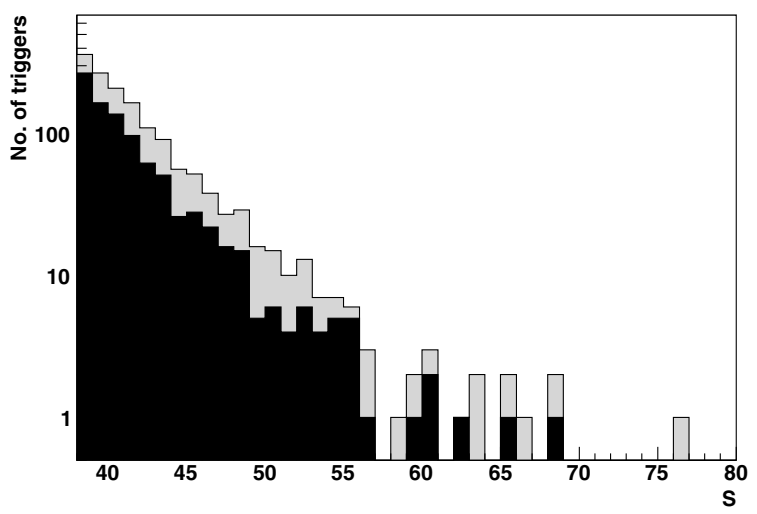

Fig. 6. Distribution of highest values for $S$ corresponding to a cut $W<$ 10 (dark) and $W<12$ (light). Cut on timer and anti-coincidence is applied in both cases.

We define the detection efficiency (DE) as the fraction of inserted pulses that is retrieved after applying the trigger conditions and the cuts that are used in the analysis. Figure 7 shows the DE for inserted pulses of strength varying from $S_{i}=36$ to $S_{i}=140$. Each pulse is inserted in the $x$-polarization and dispersed with a simTEC $=12$. The de-dispersion is done with STEC $=10$ to simulate a practically unavoidable error in the STEC value. The blue, red, and green lines in Fig. 7 show the DE for recovering pulses with strength exceeding $S_{\text {th }}>50, S_{\text {th }}>60$ and $S_{\text {th }}>70$ respectively. Due to interference with the background the recovered pule strength differs from the input value $S_{i}$. The dotted lines show the DE without any width cut applied. Solid lines represents the DE with width cut $W<8$, dashed lines show width cut $W<10$, whereas width cut $W<12$ is shown by dash-dotted lines.

From Fig. 7 it can be seen that the DE tends to saturate to unity for large pulses, as is to be expected. However one also sees that the width cut may severely limit the DE which even worsens with increasing $S_{i}$. The reason for this is that with increasing pulse strength the width (as defined in this work) increases. For really large pulses the signal may saturate causing an additional broadening of the recovered pulse. In general one also sees that the input pulse has to be about 10 units in magnitude larger that the threshold to be recovered with more than $50 \%$ efficiency.

The effect on the DE of the difference between the STEC value used in the generation of the pulse (simTEC) and the value used in the analysis is studied in Fig. 8 for pulses of strength $S_{i}=$ 80 , width cut $W<12$ and simTEC $=10$. There are two effects

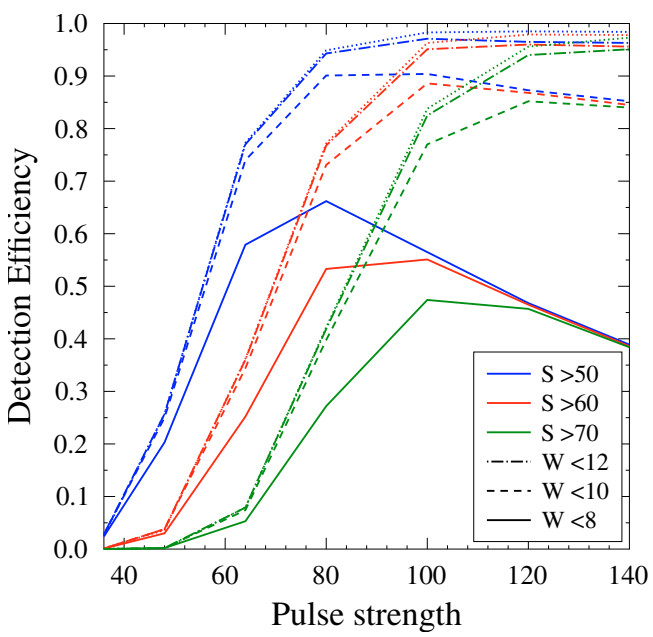

Fig. 7. The detection efficiency is shown as function of pulse strength for various settings of the trigger conditions as discussed in the text. The colors correspond to different values for the pulse-strength thresholds $S_{\text {th }}$ and the line styles correspond to different maximum widths. The dotted curve indicates the efficiency when no width cut is applied. All pulses are simulated with $\operatorname{simTEC}=12$ and analysed with TEC $=10$.

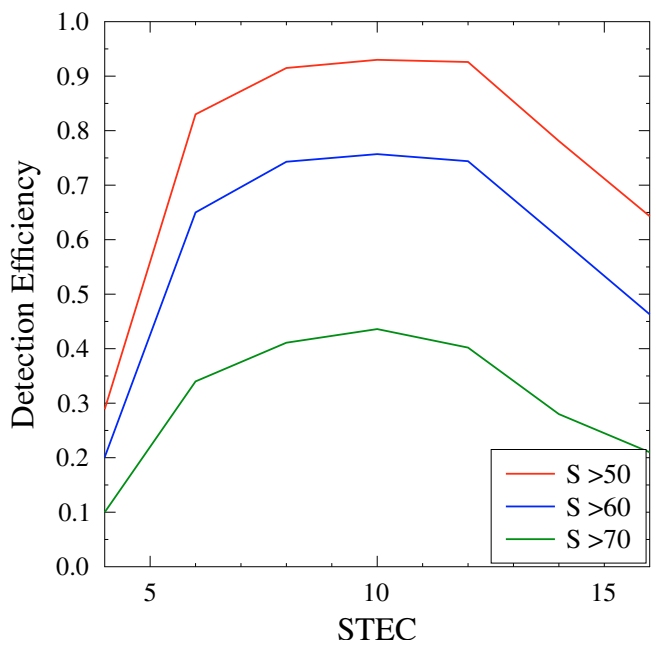

Fig. 8. The detection efficiency for pulses of strength $S_{i}=80$ and simTEC $=10$ is shown as function of the STEC value used in the analysis. The colors correspond to different thresholds.

playing a role here. Firstly, a larger error in the STEC results in a more dispersed pulse for which the power is divided over more time samples. Secondly, the range of time samples scanned in the different frequency bands after a trigger has been found in the first band, depends on the STEC value. If simTEC is much smaller than the actual STEC value, the pulse may be located outside the scanning range and is not found. When simTEC is higher than the actual value, this problem does not occur, causing an asymmetry in Fig. 8.

Due to the presence of the Earth magnetic field, the polarized radio signal is subject to a Faraday Rotation in the ionosphere (see Appendix C.2) which induces a rotation of the angle of linear polarization across the frequency band. On Earth the pulse will thus be polarized in the $x$-direction for certain frequencies while in the $y$-direction for another frequency. In each polarization direction the signal will thus cover a rather limited band width causing a broadening of the pulse and thus to a possible decrease in the DE. Figure 9 shows the DE as function of the pulse strength including the effect of Faraday rotation. A rather 


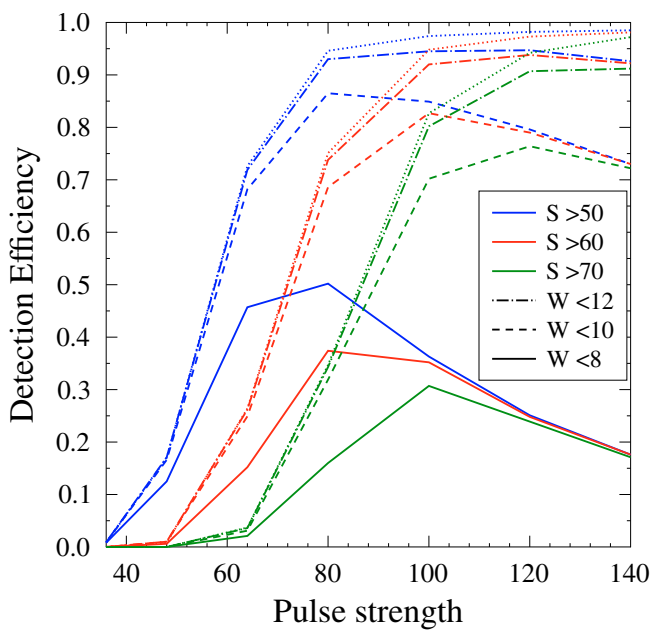

Fig. 9. Same as Fig. 7 however a Faraday rotation of the polarization direction is taken into account.

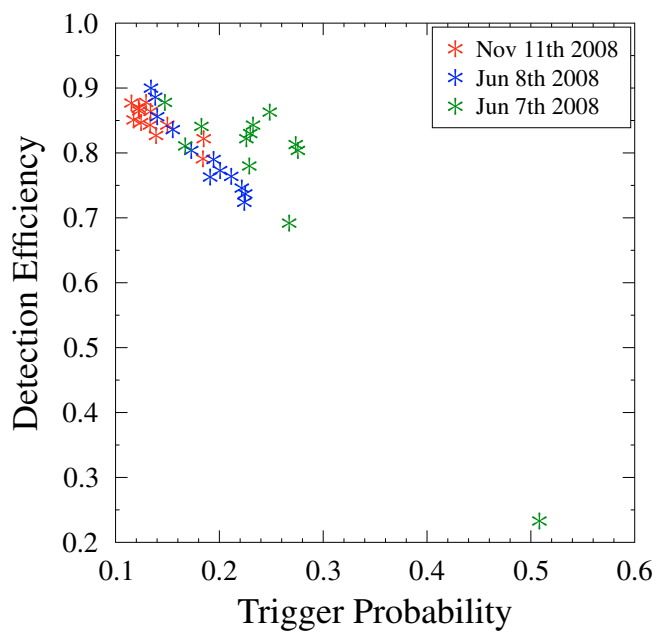

Fig. 10. The detection efficiency for pulses added with strength 25, simTEC 12, STEC 10 to different data traces of $10 \mathrm{~s}$ each is plotted vs. the number of triggers in the data trace before addition of the pulse. The colors correspond to observation runs at different dates.

large decrease in the DE is seen when width cuts are applied. This behavior is observed for all values of $S_{\text {th }}$. We therefore adopt the $W<12$ cut to be used on the analysis of the data.

We have observed a sizable dependence of the determined $\mathrm{DE}$ on the data trace which was used. First we have investigated a possible correlation of the DE value with RFI-power but this was not conclusive. We found however a very pronounced correlation between the DE and the number of raw triggers in the time trace. To show this we have determined the DE for a wide selection of raw time traces. We concentrated on the case where pulses of strength $S_{i}=100$, simTEC $=12$, STEC $=10$, were added to time traces taken from observations of June 7th 2008, June 8th 2008, and Nov. 11th 2008. We have processed 12 consecutive traces each of $10 \mathrm{~s}$. The pulses above a threshold of $S_{\text {th }}=65$ were recovered using a width cut $W<12$. We found a clear anti-correlation between the $\mathrm{DE}$ and the raw trigger rate, see Fig. 10. On the basis of this analysis the data of June 7th 2008 and June 8th 2008, have been excluded from analysis because of the large number of triggers. Other observations show a raw trigger rate of less than 40 per time trace of $0.1 \mathrm{~s}$. The observed correlation can be understood from the fact that if the algorithm finds a pulse in a spectrum, this spectrum will not be searched any further for the occurrence of another pulse. Thus if each spectrum in a $0.1 \mathrm{~s}$ time trace generates a trigger, the chance of recovering the added pulse will be vanishingly small.

\section{Effective observation time}

The effective observation time is decreased by a number of effects. After we have excluded the two observation runs which harbour exceptionally large numbers of raw triggers, we have $51.1 \mathrm{~h}$ of dual-beam observation time left. In the data analysis some files are missing or are not usable due to various reasons, such as hardware and software failures, corresponding to a loss of $3.5 \mathrm{~h}$.

When a trigger is found the rest of the time trace is not scanned for pulses. Per raw trigger this corresponds to a mean lost time of $250 \mu \mathrm{s}$ (single beam). Because of the coincidence cut, the whole time trace in the other beam should also be counted as lost time, resulting in another $500 \mu$ s (single beam). For 7.6 million raw triggers this adds up to $0.8 \mathrm{~h}$ (dual beam).

The cut on the timer pulses should be regarded as cutting out observation time, but this has already been accounted for in the previous step. Each time the system triggers on the timing pulse $750 \mu \mathrm{s}$ (single beam) is lost, as is the case for any other trigger.

After RFI removal the first and last 250 samples of a time trace have to be neglected due to FFT edge effects, corresponding to a $0.25 \%$ loss of observation time.

The total observation time is therefore $(51.1-3.5-0.8) \times$ $0.9975=46.7 \mathrm{~h}$ of dual beam data. Each beam covers about a third of the lunar area.

\section{Background}

For a radio antenna, the $1 \sigma$ noise power density $F_{\mathrm{n}}[\mathrm{Jy}]$ is given by

$$
F_{\mathrm{n}}=\frac{2 k T_{\mathrm{sys}}}{\sqrt{\Delta t \Delta v} A_{\mathrm{eff}}} 10^{26} \mathrm{Jy},
$$

where $k$ is the Boltzmann constant $\left(1.38 \times 10^{-23}\right.$ Joules $), T_{\text {sys }}$ is the antenna effective temperature in Kelvins, $\Delta t$ and $\Delta v$ are the time and frequency bins of the measurement, and $A_{\text {eff }}$ is the effective area of the telescope in $\mathrm{m}^{2}$. For the 14 Westerbork antennas we used, $A_{\text {eff }}=491 \mathrm{~m}^{2}$. For the measuring band which we need, the LFFE measuring between 113 and $170 \mathrm{MHz}$, one has a $T_{\text {sys }}$ of $400-700 \mathrm{~K}$. We use 11 antennas, yielding for the noise power per Nyquist time sample, $\Delta t \Delta v=0.5$,

$$
F_{\mathrm{n}}=\frac{2 \times 1.38 \times 10^{-23}}{11 \sqrt{0.5}} 10^{26}\left(\frac{T_{\mathrm{sys}}}{491}\right)=349\left(\frac{T_{\mathrm{sys}}}{491}\right) \mathrm{Jy}
$$

which covers the range 286-500 Jy. In the following we have adopted the value of $\sigma^{2}=400 \mathrm{Jy}$ as the average of the observing bandwidth.

\section{Results}

In $46.7 \mathrm{~h}$ of data no triggers were found with a strength exceeding $S_{\text {th }}=77$. To convert this into a probability for not observing the Moon we calculate the DE curve for a detection threshold $S_{\text {th }}=77$ including the effects of Faraday rotation, see Fig. 11. From this figure it can be seen that the DE reaches a value of $87.5 \%$ for pulses in excess of $S_{i}>120$ and width cut $W<12$. This corresponds to $120 \sigma^{2} \times 5=240 \mathrm{kJy}$. For pulses of lower 


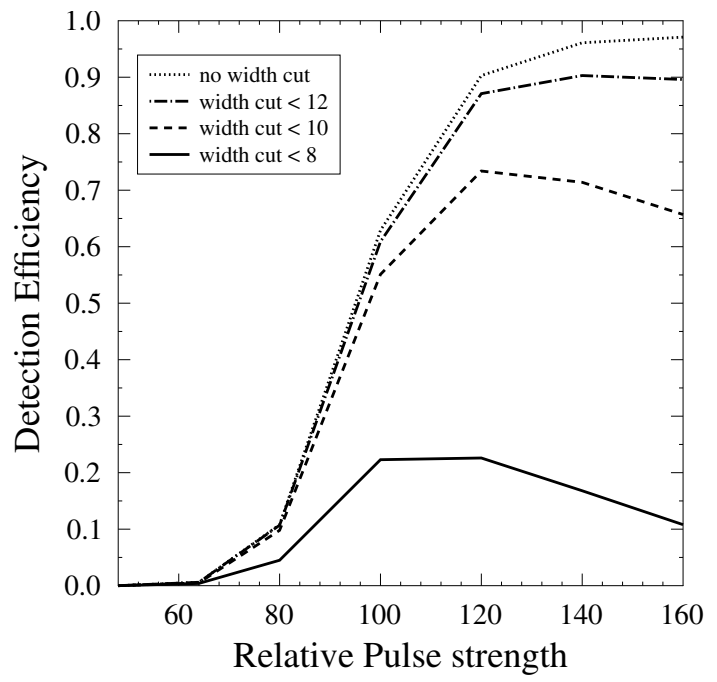

Fig. 11. The detection efficiency for a detection threshold $S_{\text {th }}=77$ is shown as a function of pulse strength for various settings of the trigger conditions as discussed in the text. The dotted curve indicates the efficiency when no width cut is applied. The effect of Faraday rotation has been taken into account.

strength the DE drops rapidly due to interference with the background. For comparison we will also consider the case for detecting pulses with a strength of $S_{i}>90$ for which the DE has dropped to $50 \%$.

The lack of pulses stronger than a certain magnitude implies a new limit on the flux of ultra-high energy neutrinos. To obtain the limit requires a calculation of the acceptance which takes into account the attenuation of the radio signal inside the Moon, the transmission at the lunar surface and the angle with respect to the arrival direction of the neutrino. On basis of the simulations which are described in Scholten (2006), the 90\% confidence level flux limit has been determined. In arriving at this the model-independent procedure described in Lehtinen et al. (2004) has been followed.

In Fig. 12 the $90 \%$ confidence limits are given for the two cases we have analyzed, $S_{i}>120$ corresponding to a $\mathrm{DE}=87.5 \%$ and $S_{i}>90(\mathrm{DE}=50 \%)$. As can be seen the gain in $\mathrm{DE}$ is far more important in setting the neutrino flux limit than the loss in sensitivity. Only at the lowest neutrino energies the result is reversed. In the rest of this work we will therefore base all arguments on the $S_{i}>120$ limit. In arriving at this limit the same assumptions have been made as in Scholten (2006), in particular that the neutrino cross sections equal the prediction given in Gandhi (2000).

The current limits in the UHE region are established by ANITA (Gorham et al. 2009) and FORTE (Lehtinen et al. 2004). Near the bottom of Fig. 12 two model predictions are plotted, the Waxman-Bahcall limit (Bahcall \& Waxman 2001) and a topdown model (Protheroe \& Stanev 1996) for exotic particles of mass $M_{X}=10^{24} \mathrm{eV}$.

Calculation of the flux of UHECRs and UHE neutrinos from the decay of topological defects is very model dependent. Parameters of such scenarios include, mass of the topological defect $M_{X}$, energy spectra, and final state composition of the decay products, and cosmological evolution of the injection rate of topological defects. The freedom provided by the reasonable range of values of these parameters is constraint by limits on the gamma ray flux at $\mathrm{GeV}-\mathrm{TeV}$ energies and neutrinos at energies above $10^{20-21} \mathrm{eV}$. The curve plotted in Fig. 12 corresponds to a $M_{X}=10^{24}$ scenario based on Protheroe \& Stanev (1996). If

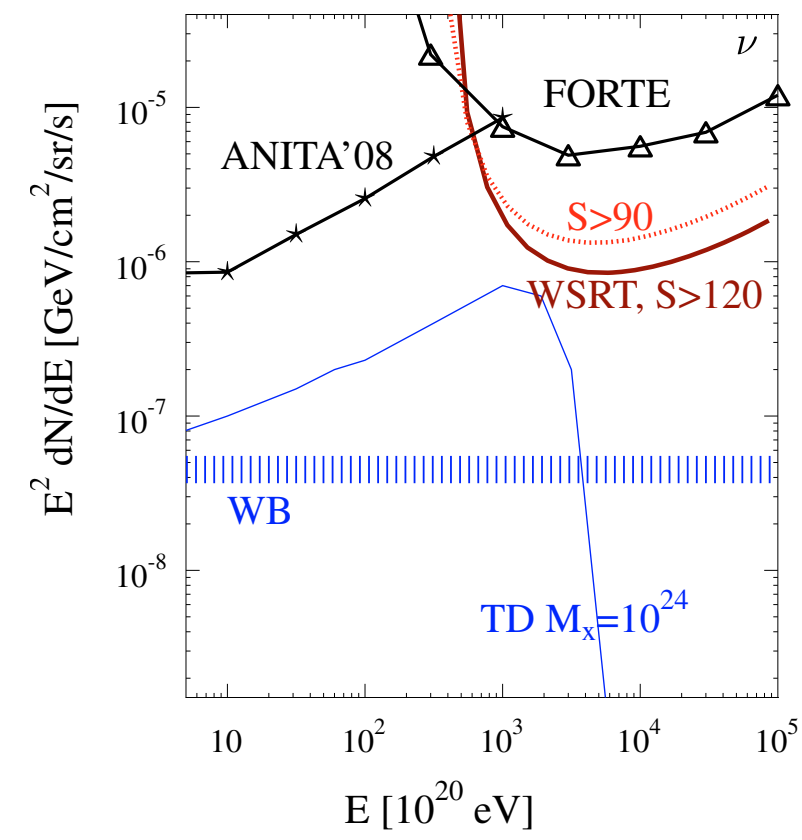

Fig. 12. Neutrino flux limit currently established with 46.7 hours of WSRT data. The brown (red) line is calculated for a minimum pulse strength of $S=120(S=90)$, corresponding to a DE of $87.5 \%(50 \%)$. Limits set by ANITA (Gorham et al. 2009) and FORTE (Lehtinen et al. 2004) are included in the plot as well as the Waxman-Bahcall flux (Waxman \& Bahcall 1998b) and a TD model prediction (Protheroe \& Stanev 1996).

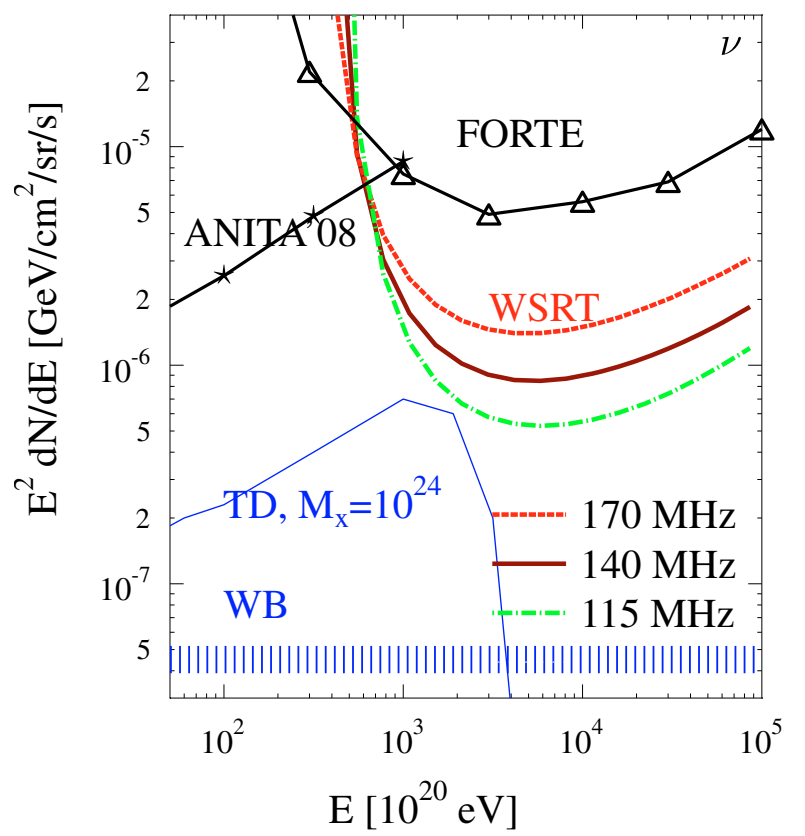

Fig. 13. Same as Fig. 12. The limits are calculated for the central frequency and the upper and lower limit of our band-width.

future limits can constrain the neutrino flux by another order of magnitude this will put constraints on the degrees of freedom of top-down models.

The acceptance calculations have been done at a frequency of $140 \mathrm{MHz}$ which is central in the observing bandwidth. Since the acceptance depends on the third power of the frequency, it varies considerably over the bandwidth as shown in Fig. 13, however the average agrees with the calculation at $140 \mathrm{MHz}$. 


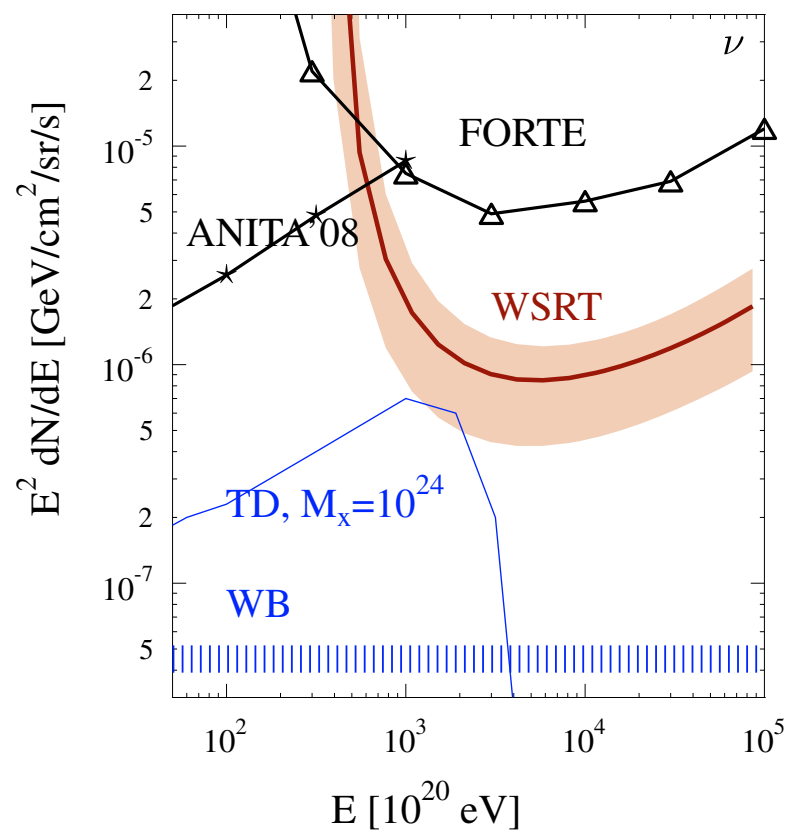

Fig. 14. [color online] Same as Fig. 12. The band shows the systematic error.

The systematic error on the acceptance is dominated by three uncertainties: the density profile, attenuation length, and stopping power of the lunar regolith. Some of these errors have been estimated in Ref. Scholten (2006). In particular the effect of density was considered which is rather complicated as an increase in the density reflects in an increased value for the index of refraction, a shorter shower length and thus a larger angular spread, more attenuation, and a lower mean neutrino interaction depth. Many of these effects appear to compensate each other resulting in an acceptance that is almost density independent, only slightly raising the minimal energy for neutrino detection. The error due to unknown variations in the density profile is therefore estimated to be $10 \%$ in threshold energy (which is the difference between the "full" and the "rock" calculation in Fig. 10 of Scholten 2006).

In Olhoeft \& Strangway (1975) the loss tangent is expressed in terms of the $\mathrm{FeO}$ and $\mathrm{TiO}_{2}$ content of the samples. On the basis of this we arrive an uncertainty in the attenuation length for radio waves of about $40 \%$ which directly reflects in a similar error in the flux determination. Also the stopping power of the regolith depends on the chemical composition where we have used a radiation length of $22.1 \mathrm{~g} / \mathrm{cm}^{2}$. An typical variation of the radiation length for the lunar regolith amounts to $0.5 \mathrm{~g} / \mathrm{cm}^{2}$. Since the angular spread is proportional to the shower length and the acceptance goes with the third power of the spread, this corresponds to a variation in the acceptance of $10 \%$. As argued before, surface roughness is not very important at our wavelength and may contribute not more than $10 \%$ to the uncertainty in determining the flux. The error in the Moon coverage of the two beams is estimated at $20 \%$. Adding these errors in quadrature gives a systematic error on the flux of $50 \%$ as indicated in Fig. 14.

From the non-observation of short radio pulses coming from the Moon, limits can also be set on the flux of UHE cosmic rays. This will be discussed in a future article as special attention has to be devoted to the calculation of the formation length for Cherenkov radiation which is important for a shower that is close to the lunar surface.
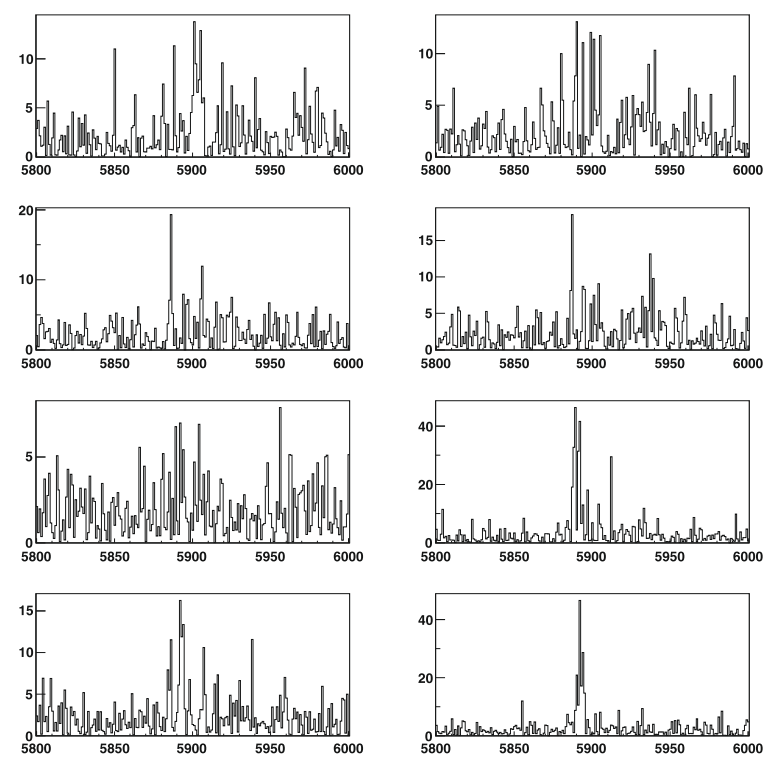

Fig. 15. Time traces of the power (polarizations added) for a typical large pulse seen in the data after applying the cuts. The P5 values are plotted for all bands (top: highest frequency, bottom: lowest frequency) and both beams (left and right). The horizontal axis displays bin number (bin size is $25 \mathrm{~ns}$ ). The power on the vertical axis is expressed in mean P5 value, with the trigger level at 5 for all bands. A trigger was only found for the right beam.
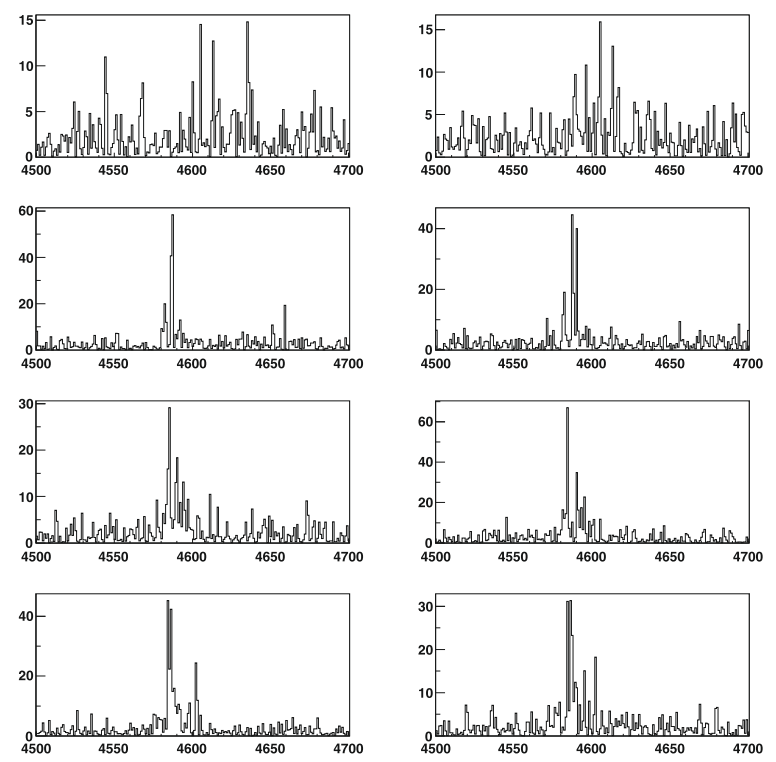

Fig. 16. Like Fig. 15, different pulse. The similar features in both beams exclude the pulse as a proper lunar pulse candidate.

\section{Discussion of the large peaks}

We have investigated the nine strongest pulses that survive the applied cuts. Figures 15 and 16 are typical examples of the time traces of such pulses. The pulses are from different observation runs and their $P 5$ values are plotted as a function of bin number (bin size is $25 \mathrm{~ns}$ ) for all frequency bands and both beams. At this stage the RFI has already been mitigated and the signal has been de-dispersed.

For both example events, the trigger was found in the righthand beam. For the event in Fig. 15 the maximum P5 value increases with decreasing frequencies. This could be due to a 
stronger signal at lower frequencies or an increase of pulsed background at higher frequencies (remember that the $P 5$ value is normalized over a $500 \mu$ s time trace for each individual band). Although the signal is clearly much smaller in the left beam, it should be noted that three out of four bands actually have a pulse that exceeds trigger level $(P 5>5)$. The event displayed in Fig. 16 has a strong signal in both beams and the only reason this event was not discarded by the anti-coincidence criterion is that the highest frequency band has a very small signal-to-noise ratio. In this band, the signal is suppressed and happens to be just above threshold in the right-hand beam but below threshold in the left-hand beam. This way, strong temporary increases in background radiation are responsible for several of the largest events that pass our criteria.

Although the event in Fig. 15 has a curious dependence on frequency, it has the properties of a proper lunar pulse of the type we are looking for, which are: i) present in all frequency bands, ii) strong polarization, iii) short after dispersion correction, and iv) present in one beam only. In order to study the possibility of the pulse to originate from the Moon we can impose an additional condition that the Faraday rotation of the polarization is of the correct magnitude.

The Faraday rotation going through a plasma with STEC $=5$ in the Earth magnetic field is about $\pi / 4$ radian over $30 \mathrm{MHz}$ (corresponding to the difference in the centroids of bands 1 and 3) at the frequencies of interest for the present study. For a pulse fully polarized in the $x$-direction in the center of band 1 one would thus expect about equal strength in the $x$ and $y$ polarization for band 3 . We have examined whether or not the ratio between the pulse strengths in the $x$ and $y$ polarization in the different frequency bands corresponds to the ratios expected on the basis of the STEC value. This criterion disqualifies the pulse in Fig. 15 as originating from outside the ionosphere.

We have studied the nine strongest pulses with $S>62$ and found that all of them are unlikely to come from the Moon, because they either have a strong signal in both beams or do not have the frequency dependent behavior expected from Faraday rotation. As a result we can safely state that we see no pulses originating from a particle cascade in the Moon with a strength larger than $S=62$. Because this analysis is done a posteriori, the threshold used for the determination of the neutrino flux limit is kept at $S=77$. In future studies, additional cut criteria based on temporary power surges in the background and Faraday rotation of the signal in the ionosphere can be implemented to further understand and reduce the background.

\section{Outlook}

The next phase in the NuMoon experiment will be to use LOFAR, the Low Frequency Array (Falcke et al. 2006), that is under construction in the Netherlands. LOFAR is a network of low frequency omni-directional radio antennas communicating over a fiber optics network. It will feature two types of antennas operating at different frequencies, the Low Band (LB) antennas cover a band of $30-80 \mathrm{MHz}$ while the High Band (HB) antennas cover the regime $110-240 \mathrm{MHz}$. The latter will be used for the NuMoon observations. LOFAR is organized in 35 stations each containing $48 \mathrm{LB}$ and $96 \mathrm{HB}$ antennas. Half of the stations are located inside the $2 \times 2 \mathrm{~km}$ core with a total collecting area of $\sim 0.05 \mathrm{~km}^{2}$. Multiple beams can be formed to cover the surface of the Moon, resulting in a sensitivity that is about 25 times better than the WSRT (Singh et al. 2008).
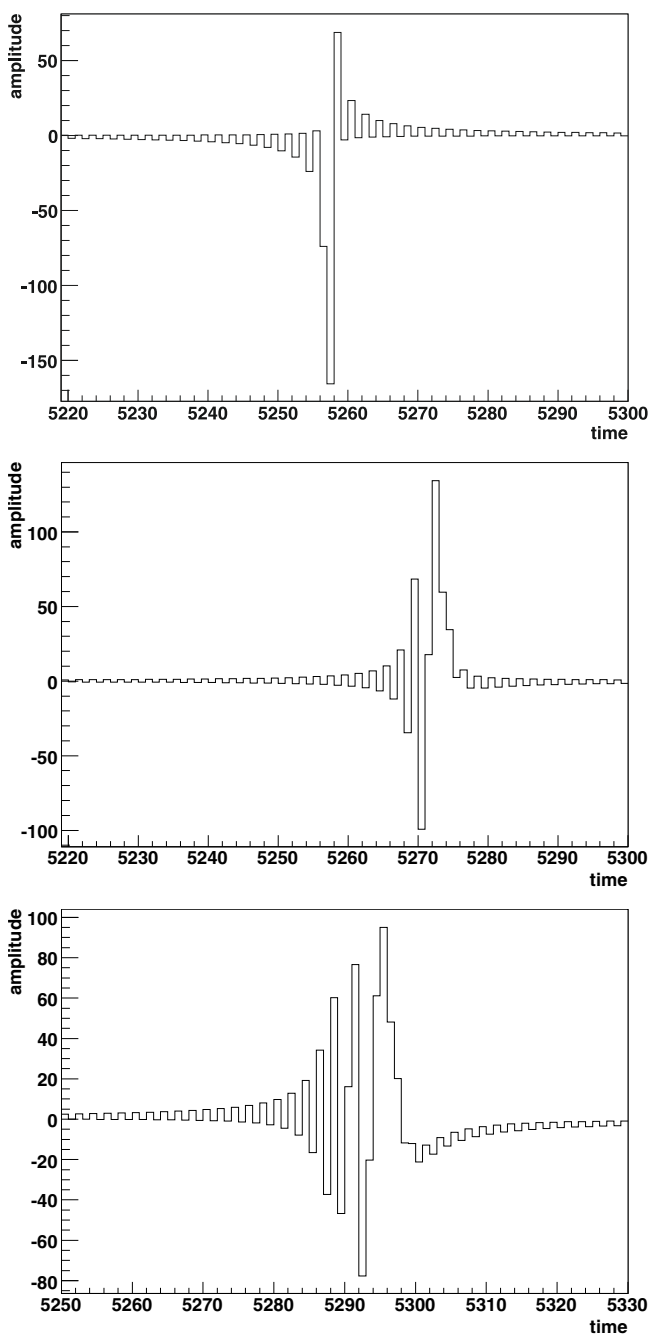

Fig. A.1. Top: time sampling of a delta peak pulse at time 5257.96 with phase $1.17 \pi$. Middle: same pulse, dispersed with TEC value 4. Bottom: same pulse dispersed with TEC value 10 .

\section{Appendix A: Bandwidth limited pulses}

To give an idea of the behaviour of bandwidth limited, Nyquist sampled pulses we show the sampling of pulse with the shape of a delta peak. Like in our analysis we take a time trace of 20000 timebins, where each timebin is $25 \mathrm{~ns}$ wide. We add a pulse at a random location with a random phase by setting the amplitude of all frequency bins to the same positive value and the phase $\Phi_{i}$ of the frequency bin $i$ to

$$
\Phi_{i}=\Phi_{0}+2 \pi i\left[1-\left(\frac{t}{20000}\right)\right]
$$

where $\Phi_{0}$ is a random phase and $t$ is a random time between 0 and 20000 . The shape of the pulse in time domain is found by performing a reverse FFT. For $\Phi_{0}=0$ and an integer value of $t$, all timebins are zero except timebin $t$, which contains a positive value. For a random $\Phi_{0}$ or a non-integer value of $t$ the pulse has a complicated shape in the time domain.

The top panel of Fig. A.1 shows how such a pulse typically spreads out over many timebins. In most cases the bulk of the pulse power is inside 2 or 3 timebins. When the pulse is dispersed the power is spread out over even more timebins. The middle and bottom panel show the pulse broadening for TEC values of 4 resp. 10. In our analysis we have defined the width of the pulse as the number of consecutive $P_{5}$ values that exceed the 
threshold. It should be noted that this differs in general from the actual width of the pulse. For example, a very large amplitude in one single timebin can give 5 consecutive $P_{5}$ triggers, while the same pulse spread out over 5 timebins will maybe produce only one threshold exceeding $P_{5}$ value.

\section{Appendix B: Statistics}

We carry out a statistical analysis to establish the expected amount of triggers and the distribution of $S$, the sum of the $P_{5}$ values over 4 frequency bands, for Gaussian background noise. When the amplitudes follow a Gaussian distribution, the probability density of the power is a chi-squared distribution

$P(x, k)=\frac{x^{k / 2-1} \mathrm{e}^{-x / 2}}{2^{k / 2} \Gamma(k / 2)}$,

where $x$ is the power in units of the standard deviation $\sigma$ and $k$ is the number of degrees of freedom. The trigger condition for a single band is $P_{5}>5$, where $P_{5}$ is given by Eq. (4). For Gaussian noise with the same standard deviation $\sigma$ in both polarizations this condition becomes

$P_{5}=\frac{\sum_{10 \text { bins }} x}{\left\langle\sum_{5 \text { bins }} x\right\rangle}=\frac{1}{5} \sum_{10 \text { bins }} x>5$,

or

$\sum_{10 \text { bins }} x>25$

The trigger chance for a single frequency band is therefore

$P_{\text {trigger }}=\int_{25}^{\infty} P(x, k=10) \mathrm{d} x \approx 0.00535$.

Consecutive $P_{5}$ values have 8 overlapping timebins (4 in both polarizations) and we have to distinguish the chance to find a trigger that comes after another trigger, $P_{\mathrm{xx}}$, and a trigger after a non-trigger, $P_{\mathrm{ox}}$. A trigger will be found after a non-trigger if:

- the eight overlapping bins add up to less than the trigger value by a certain value $A$;

- the two bins for the non-trigger add up to a value smaller than A;

- the two bins for the trigger add up to a value larger than $A$.

To find the total probability we integrate over all possible values of $A$

$$
\begin{aligned}
P_{\mathrm{ox}}= & \int_{0}^{25} P(25-A, k=8)\left[\int_{0}^{A} P(x, k=2) \mathrm{d} x\right] \\
& \times\left[\int_{A}^{\infty} P(x, k=2) \mathrm{d} x\right] \mathrm{d} A \\
\approx & 0.00283 \approx 0.53 P_{\text {trigger }} .
\end{aligned}
$$

The chance of finding a trigger after another trigger is

$P_{\mathrm{xx}}=P_{\text {trigger }}-P_{\mathrm{ox}} \approx 0.00252 \approx 0.47 P_{\text {trigger }}$,

so about half of the triggers is found clustered together which has consequences for our analysis.

For a complete trigger the trigger condition has to be met in all 4 frequency bands. In bands 2,3 , and 4 a range of $P_{5}$ values will be scanned based on the STEC value. Suppose in the first
Table B.1. Predicted and simulated trigger chance.

\begin{tabular}{llll}
\hline \hline STEC & $\left(N_{2}, N_{3}, N_{4}\right)$ & Pred. chance & Sim. chance \\
\hline 5 & $(3,5,7)$ & $1.5 \times 10^{-8}$ & $1.5 \times 10^{-8}$ \\
10 & $(5,7,11)$ & $3.6 \times 10^{-8}$ & $3.8 \times 10^{-8}$ \\
15 & $(5,9,15)$ & $6.3 \times 10^{-8}$ & $6.0 \times 10^{-8}$ \\
\hline
\end{tabular}

band a trigger is found after a non-trigger. The chance to find a trigger in band no. $i$ is

$P_{i}=P_{\text {trigger }}+\left(N_{i}-1\right) \cdot P_{\mathrm{ox}}+O\left(P_{\text {trigger }}^{2}\right)$,

where $N_{i}$ is the number of values scanned in band $i$. Terms of the order of $P_{\text {trigger }}^{2}$ arise from properly adding the chances of finding a trigger in one of the $N_{i}$ bins and deviations in the chance of finding a trigger in a certain bin depending on the number of bins without a trigger than precede it. The chance to find a trigger in the three upper bands is $P_{2} \cdot P_{3} \cdot P_{4}$ for this case.

When, however, the trigger in band 1 came after another trigger in band 1, the chance to find a trigger in the three upper bands is smaller because we know that for the previous trigger in band 1, not all upper bands had a trigger. If that were the case the pulse search would have skipped the rest of the time trace. The range of timebins that is scanned in the upper bands is overlaps with scan after the previous trigger in band 1. Actually, in each band only one new timebin is scanned. The chance to find a trigger in the upper bands is therefore now reduced to $P_{2} \cdot P_{3} \cdot P_{4} \cdot\left(1-P_{\text {reduce }}\right)$, where $P_{\text {reduce }}$ is the chance that there was also a trigger in the previous scan. To not have a trigger in the previous scan, at least one of bands should have the trigger in the last timebin, because this is the only bin that is unique for the new scan. In other words, the previous scan also had a trigger when for each upper band the trigger is located in any but the last scanned timebin. The chance for the trigger to be in the last timebin of band $i$ is $P_{\mathrm{ox}} / P_{i}$, so we find

$P_{\text {reduce }}=\left(1-\frac{P_{\mathrm{ox}}}{P_{2}}\right)\left(1-\frac{P_{\mathrm{ox}}}{P_{3}}\right)\left(1-\frac{P_{\mathrm{ox}}}{P_{3}}\right)+O\left(P_{\text {trigger }}\right)$,

where we neglect terms of order $P_{\text {trigger }}$ that arise from the possibility that a trigger is in the last timebin, but also in the first timebin of the previous scan. The complete chance of finding a trigger in all four bands is given by

$P_{4 \text { trig }}=P_{\mathrm{ox}} P_{2} P_{3} P_{4}+P_{\mathrm{xx}} P_{2} P_{3} P_{4}\left(1-P_{\text {reduce }}\right)+O\left(P_{\text {trigger }}^{5}\right)$.

In Table B. 1 the values of $P_{4 \text { trig }}$ are given for different STEC values. In the calculation higher order terms are incorporated. The rightmost column shows the simulated trigger chance that is found by applying our data analysis code on generated Gaussian noise. In the code the Gaussian noise is rounded off to the nearest of the 34 values that are part of the dynamic range of the PuMa-II system. This is the main reason for the discrepancy between analytic predictions and the simulation results, which is smaller than $5 \%$.

To arrive at the (not normalized) probability distribution of $S$, we take 4 probability distributions of values $a$ through $d$, and integrate to find the distribution of $x=a+b+c+d$

$$
\begin{aligned}
& P(x)=\int_{25}^{x-75} \mathrm{~d} a \int_{25}^{x-a-50} \mathrm{~d} b \int_{25}^{x-a-b-25} \mathrm{~d} c P(c, k=10) \\
& \quad \times P(b, k=10) P(a, k=10) P(x-a-b-c, k=10),
\end{aligned}
$$

where the limits of the integral are chosen in such a way that $a, b, c$ and $d$ all exceed 25 individually. By normalizing the distribution $P(x)$ with the total amount of triggers projected with 
Eq. (B.9) and substitute $S=x / 5$ (see Eq. (B.2)), we arrive at the analytical noise background prediction that is plotted in Fig. 4.

\section{Appendix C: lonospheric effects}

The ionosphere is a plasma where the density of free electrons affects the propagation of electromagnetic waves which may show as a dispersion of the signal or a frequency-dependent rotation of the linear polarization.

\section{C.1. Dispersion}

As the radio signal propagates through the Earth's ionosphere it is dispersed by

$\phi(v)=2 \pi \int \mathrm{d} z v\left(\sqrt{1-\frac{v_{\mathrm{p}}^{2}}{v^{2}}}-1\right) / c$,

where $\Delta \phi$ is the phase shift at frequency $v$. The integral is taken over the traversed distance $z, c$ is the speed of light in vacuum, and $v_{\mathrm{p}}$ is the the plasma frequency

$v_{\mathrm{p}}^{2}=\frac{n_{\mathrm{e}} e^{2}}{4 \pi^{2} \epsilon_{0} m_{\mathrm{e}}}=8.07 \times 10{ }^{17} \mathrm{STEC} / \Delta z$,

where $n_{\mathrm{e}}$ is the electron number density, $e$ the elementary charge, $\epsilon_{0}$ the permittivity of vacuum, and $m_{\mathrm{e}}$ the electron mass. For the ionosphere $v_{\mathrm{p}} \approx 3 \mathrm{MHz}$ and

$$
\int \frac{v_{\mathrm{p}}^{2}}{2 c} \mathrm{~d} z=1.34 \times 10^{9} \mathrm{STEC},
$$

where we use the Slanted Total Electron Content (STEC), which is the electron density integrated along the distance the pulse has traveled through the ionosphere. The STEC is given in TEC units (TECU) where 1 TECU $=10^{16}$ electrons $/ \mathrm{m}^{2}$. The phase shift is approximately

$\phi(v) \approx 2 \pi \frac{1.34 \times 10^{9} \mathrm{STEC}}{v}$,

corresponding to a time offset of

$\Delta t=1.34 \times 10^{9} \cdot \operatorname{STEC}\left(\frac{1}{v_{1}^{2}}-\frac{1}{v_{2}^{2}}\right)$,

between two frequency components $v_{1}$ and $v_{2}$. For an interval of $20 \mathrm{MHz}(140-160 \mathrm{MHz})$ and STEC $=10$ the difference in time delay is $\Delta t \approx 1.6 \times 10^{-7} \mathrm{~s}$, which corresponds to 6.4 time samples for a $40 \mathrm{MHz}$ sampling frequency.

\section{C.2. Faraday rotation}

In the presence of a magnetic field the linear polarization direction of an electromagnetic signal will rotate over a finite angle. This Faraday rotation is usually expressed in terms of a rotation measure (RM)

$\beta_{\mathrm{F}}=\mathrm{RM} \lambda^{2}=\mathrm{RM} c^{2} / v^{2}$.
In units of radians per square meter $\left(\mathrm{rad} / \mathrm{m}^{2}\right), \mathrm{RM}$ is calculated as

$$
\begin{aligned}
\mathrm{RM} & =\frac{e^{3}}{8 \pi^{2} \varepsilon_{0} m^{2} c^{3}} \int_{0}^{d} n_{\mathrm{e}} B \mathrm{~d} s=2.62 \times 10^{-13} \int_{0}^{d} n_{\mathrm{e}} B \mathrm{~d} s \\
& =2.62 \times 10^{3} \times \mathrm{STEC} \times B_{\|}
\end{aligned}
$$

with $B$ in teslas (T), and $n_{\mathrm{e}}$ in $\mathrm{m}^{-3}$.

The difference in the Faraday rotation angle for two frequency components $v_{1}$ and $v_{2}$ can be related to the difference in time delay as $\Delta \beta_{\mathrm{F}}=\frac{e B}{m_{\mathrm{e}} c} c \Delta t=5.27 \times 10^{6} \Delta t$. For an interval of $20 \mathrm{MHz}(140-160 \mathrm{MHz})$ and an STEC = 10 [tecu] we obtain for the difference in Faraday rotation angles

$\Delta \beta_{\mathrm{F}}=3 \times 10^{8} / 50 \times \Delta t=0.96$

which is appreciable.

\section{References}

Abraham, J. E. A. 2007, Science, 318, 938

Abraham, J. E. A. 2008, Phys. Rev. Lett., 101, 061101

Ahrens, J. E. A. 2003, Nucl. Phys. Proc. Suppl., 118, 388

Alvarez-Muñiz, J., \& Zas, E. 1997, Phys. Lett. B, 411, 218

Alvarez-Muñiz, J., \& Zas, E. 1998, Phys. Lett. B, 434, 396

Askaryan, G. 1962, Sov. Phys., JETP, 14, 441

Aslanides, E. E. A. 1999 [arXiv: astro-ph/9907432]

Bahcall, J., \& Waxman, E. 2001, Phys. Rev. D, 64, 64

Barwick, S. E. A. 2006, Phys. Rev. Lett., 96, 171101

Beresnyak, A., Dagkesamanskii, R., Zheleznykh, I., Kovalenko, A., \& Oreshko, V. 2005, Astron. Rep., 49, 127

Dagesamanskii, R., \& Zheleznyk, I. 1989, Sov. Phys. JETP, 50, 233

Falcke, H., \& Gorham, P. 2003, Astropart. Phys., 19, 477

Falcke, H. D. E., et al. 2006, Long Wavelength Astrophysics, 26th meeting of the IAU, Joint Discussion 12, 21 August 2006, Prague, Czech Republic, JD12, \#16, 12

Gandhi, R. 2000, Nucl. Phys. B, 91, 453

Gorham, P. E. A. 2000, Phys. Rev. E, 62, 8590

Gorham, P. E. A. 2004, Phys. Rev. Lett., 93, 41101

Gorham, P. W., Allison, P., Barwick, S. W., et al. 2009, Phys. Rev. Lett., 103, 051103

Greisen, K. 1966, Phys. Rev. Lett., 16, 748

Hankins, T., Ekers, R., \& O’Sullivan, J. 1996, MNRAS, 283, 1027

Heiken, G., Vaniman, D., \& French, B. 1991, Lunar Sourcebook, A users guide to the Moon (Cambridge Univ. Press)

James, C., \& Protheroe, R. 2009, Nucl. Instrum. Meth. Phys. Res. A, 604, S222 James, C. W., Ekers, R. D., Alvarez-Muniz, J., et al. 2009, Nucl. Instrum. Meth. Phys. Res. A, 604, S112

Jansen, G. E. A. 2009, A\&A, 498, 223

Karuppusamy, R., Stappers, B., \& Van Straten, W. 2008 [arXiv: astro-ph/0802.2245]

Katz, U. E. A. 2006, Nucl. Instrum. Meth. A, 567, 457

Lehtinen, H., Gorham, P., Jacobson, A., \& Roussel-Dupre, R. 2004, Phys. Rev. D, 69, 013008

Olhoeft, G., \& Strangway, D. 1975, Earth Plan. Sci. Lett., 24, 394

Protheroe, R., \& Stanev, T. 1996, Phys. Rev. Lett., 77, 3708

Saltzberg, D. E. A. 2001, Phys. Rev. Lett., 86, 2802

Scholten, O., Buitink, S., Bacelar, J., et al. 2009, Phys. Rev. Lett., 103, 191301

Scholten, O. E. A. 2006, Astropart. Phys., 26, 219

Singh, K., et al. 2008, Proc. of 21th ECRS

Stanev, T. 2004 [arXiv: astro-ph/0411113]

Waxman, E., \& Bahcall, J. N. 1998a, in 19th Texas Symposium on Relativistic Astrophysics and Cosmology, ed. J. Paul, T. Montmerle, \& E. Aubourg

Waxman, E., \& Bahcall, J. N. 1998b, Phys. Rev. D, 59, 023002

Wieczorek, M., \& Zuber, T. 2001, Geophys. Res. Lett., 28, 4023

Zas, E., Halzen, F., \& Stanev, T. 1992, Phys. Rev. D, 45, 362

Zatsepin, G., \& Kuzmin, V. 1966, Pis'ma Zh. Eksp. Teor. Fiz., 4, 114 